Investigations of Magnetic Overlayers at the Advanced Photon Source

J. G. Tobin, S. Yu, M. T. Butterfield

June 29, 2009

Journal of Vacuum Science and Technology A 
This document was prepared as an account of work sponsored by an agency of the United States government. Neither the United States government nor Lawrence Livermore National Security, LLC, nor any of their employees makes any warranty, expressed or implied, or assumes any legal liability or responsibility for the accuracy, completeness, or usefulness of any information, apparatus, product, or process disclosed, or represents that its use would not infringe privately owned rights. Reference herein to any specific commercial product, process, or service by trade name, trademark, manufacturer, or otherwise does not necessarily constitute or imply its endorsement, recommendation, or favoring by the United States government or Lawrence Livermore National Security, LLC. The views and opinions of authors expressed herein do not necessarily state or reflect those of the United States government or Lawrence Livermore National Security, LLC, and shall not be used for advertising or product endorsement purposes. 


\title{
Investigations of Magnetic Overlayers at the Advanced Photon Source
}

\author{
J.G. Tobin*, S.-W. Yu, M.T. Butterfield, \\ Lawrence Livermore National Laboratory, LLNS-LLC, Livermore, CA \\ Takashi Komesu", and G.D. Waddill, \\ Missouri University of Science and Technology, Physics Department, Rolla, MO
}

\begin{abstract}
Magnetic overlayers of $\mathrm{Fe}$ and Co have been investigated with X-ray Magnetic Circular Dichroism in X-ray Absorption Spectroscopy (XMCD-ABS) and Photoelectron Spectroscopy (PES), including Spin- Resolved Photoelectron Spectroscopy (SRPES), at Beamline 4 at the Advanced Photon Source (APS). Particular emphasis was placed upon the interrogation of the $2 p$ levels of the Fe. PACS numbers

73.90.+f, 75.25.+z, 79.60.-i

*Corresponding Author, Tobin1@LLNL.Gov \#Present address: Spring8, Japan Synchrotron Radiation Research Institute, (JASRI), 1-1-1, Kouto, Sayo-cho, Sayo-gun, Hyogo 679-5198 Japan
\end{abstract}

\section{Introduction}

Over the course of several years, we have pursued the investigation of magnetic overlayers with a variety of electron spectroscopies at different synchrotron radiation facilities. [1-7] Over time, it became apparent that the most demanding experiment, the double polarization photoelectron spectroscopy or "complete experiment," could best attempted at Beam-line 4 at the Advanced

Tobin, LLNL

Page 1

$6 / 25 / 09$

J. Vac. Sci. Tech., AVS International Symposium, San Jose, CA, USA, 2009 


\section{Investigations of Magnetic Overlayers at the Advanced Photon Source}

Photon source. [8] By combining the circularly polarized x-rays from the undulator at Beam-line 4 with the spin resolving capabilities of our Spin Spectrometer [9], it would be possible to pursue the double polarization experiment upon the $2 p$ levels of $3 d$ magnetic materials such as $\mathrm{Fe}$ and $\mathrm{Co}$.

\section{Experimental}

Our spin-resolved photoemission spectroscopic spectrometer has been previously described [9]. The photo-excited electrons are collected in a hemispherical electron energy analyzer (Physical Electronics Model 3057) with multi-channel electron detection. (Figure 1) In the Mini-Mott, spin polarized electron detector, the electrons are accelerated to $\sim 25 \mathrm{kV}$, with four channeltrons positioned horizontally and vertically used for electron counting. For the measurements described here, the incident angle was 55 degrees off the surface normal (in the yz plane) and the photoelectrons were collected at normal emission. All magnetic SRPES measurements were made in remanance, with the absence of an applied field during the SRPES data collection. The Cu crystal was cleaned with $\mathrm{Ar}^{+}$sputtering followed by annealing to about $450{ }^{\circ} \mathrm{C}$. All measurements were made on Beam-line 4ID-C at the Advanced Photon Source at Argonne National Laboratory [8].

\section{Results and Discussion}

Ultra-thin film preparation is a crucial step in these experiments. Ultra-thin films of $\mathrm{Fe}$ and $\mathrm{Co}$ were grown on $\mathrm{Cu}(100)$ as in previous studies [1-7]. The films exhibited a $p(1 \times 1)$ LEED pattern and no observable contamination as judged 


\section{Investigations of Magnetic Overlayers at the Advanced Photon Source}

from X-ray Photoemission Spectroscopy (XPS) measurements, as can be seen in Figures 2 and 3 . Figures 2 and 3 also provide examples of how the film growth and contamination was followed using XPS. Obviously, the $\mathrm{Fe}, \mathrm{Co}, \mathrm{Cu}$ and Oxygen core levels can all be isolated and monitored separately.

Independently of the SRPES experiment, the magnetic character of the films of Fe and Co was determined using x-ray magnetic circular dichroism in $\mathrm{x}$ ray absorption spectroscopy. [1,6] As can be clearly seen in Figures 4 and 5 , strong dichroic effects are observed for both the Fe and Co, before and after the SRPES measurements. (In the plots of Figures 4 and 5, we show the uncorrected photon energy. Oftentimes, monochromators can display offsets and skewing, that vary with time. Even without correction, it is clear that these are the appropriate assignments. The correct edge energies are shown in the insets, for comparison. This effect is also illustrated in Figure 2, with the PES data.)

Ultimately, the goal was the performance of the double polarization experiment upon the $2 p$ levels. An example of our data is shown in Figure 6. There is observation of a strong polarization and a well-behaved Instrumental Asymmetry. [10] The detailed analysis of this spectrum will be presented elsewhere. [11]

Finally, we have developed a simple single electron picture that can mimic the fundamental physical effects of the double polarization experiment. $[7,11]$ Examples of its application to a number of limiting cases are shown in Figure 7.

Tobin, LLNL

Page 3

$6 / 25 / 09$

J. Vac. Sci. Tech., AVS International Symposium, San Jose, CA, USA, 2009 


\section{Investigations of Magnetic Overlayers at the Advanced Photon Source}

Here, we have utilized an idealized configuration with a strong spectroscopic effect, then used various magnetic and spin-orbit splittings to illustrate the trends to be expected for the elemental series $\mathrm{Fe}, \mathrm{Co}, \mathrm{Ni}$ and $\mathrm{Cu}$. Three Fano or dichroic cases have been considered: strong positive, zero and strong negative. These dichroic effects can change as a function of photon energy, thus the need to consider the three cases. [7] For $\mathrm{Cu}$, where there is no magnetism, only the spinorbit driven effects will be observed. As expected, for the zero dichroism case, the Cu spin up and spin down spectra are identical and the ratio is identically one. For the strong dichroism cases ( $c=+/-0.5)$, the retention of the dichroism can be seen as the exchange splitting grows progressively from $\mathrm{Ni}$ to $\mathrm{Co}$ to $\mathrm{Fe}$. Interestingly, our experimental result for Fe looks like the zero dichroism case and this is not unexpected. In that experiment, the strong magnetic moment and the experimental geometry converge to miminize the impact of the circularly polarized x-rays. The detailed discussion of this result is presented elsewhere. [11]

\section{Summary}

We have successfully carried out double polarization photoelectron spectroscopy of $\mathrm{Fe} / \mathrm{Cu}(001)$ at Beam-line 4 of the Advanced Photon Source. Supporting measurements as well as the results of a single electron model were presented and briefly discussed.

\section{Acknowledgments}

Lawrence Livermore National Laboratory is operated by Lawrence Livermore National Security, LLC, for the U.S. Department of Energy, National Nuclear Security Administration under Contract DE-AC52-07NA27344. This work Tobin, LLNL 


\section{Investigations of Magnetic Overlayers at the Advanced Photon Source}

was supported by the DOE Office of Basic Energy Science and Campaign 2/WCI/LLNL. The APS has been built and operated under funding from the Office of Basic Energy Science at DOE. We would like to thank the scientific and technical staff of Sector 4 of the Advanced Photon Source for their technical assistance in supporting this work. TK would like to thank Prof. Peter A. Dowben at University of Nebraska-Lincoln for valuable scientific discussions.

\section{References}

1. J.G. Tobin, G.D. Waddill and D.P. Pappas, Phys. Rev. Lett. 68, 3642 (1992);

J.G. Tobin, G.D. Waddill, A.F. Jankowski, P.A. Sterne, and D.P. Pappas, Phys. Rev. B1 52, 6530 (1995).

2. G.D. Waddill, J.G. Tobin and D.P. Pappas, Phys. Rev. B, Rapid Comm. 46, 552 (1992).

3. G.D. Waddill, J.G. Tobin, X. Guo, and S.Y. Tong, Phys. Rev. B 50, 6774 (1994).

4. J.G. Tobin et al, Surf. Rev. Lett. 3, 1429 (1996).

5. F.O. Schumann, R.F. Willis, K.W. Goodman, and J.G. Tobin, Phys. Rev. Lett. 79, 5166 (1997).

6. J. G. Tobin et al, J. Vac. Sci. Tech., B 14, 3171 (1996); S.Z. Wu et al, J. Vac. Sci. Tech. A15, 2287 (1997).

7. J.G. Tobin and F.O. Schumann, Surface Science 478, 211 (2001).

8. J.W. Freeland et al, Rev. Sci. Instrum. 73, 1408 (2002).

9. J.G. Tobin et al, Nucl. Instrum. Methods A582, 162 (2007).

10.S.W. Yu et al, submitted to RSI, 2009.

11. T. Komesu et al, submitted to JPCM, 2009.

12. X-ray Data Handbook, A. Thompson et al, LBNL, Jan 2001.

Tobin, LLNL

Page 5

$6 / 25 / 09$

J. Vac. Sci. Tech., AVS International Symposium, San Jose, CA, USA, 2009 


\section{Investigations of Magnetic Overlayers at the Advanced Photon Source}

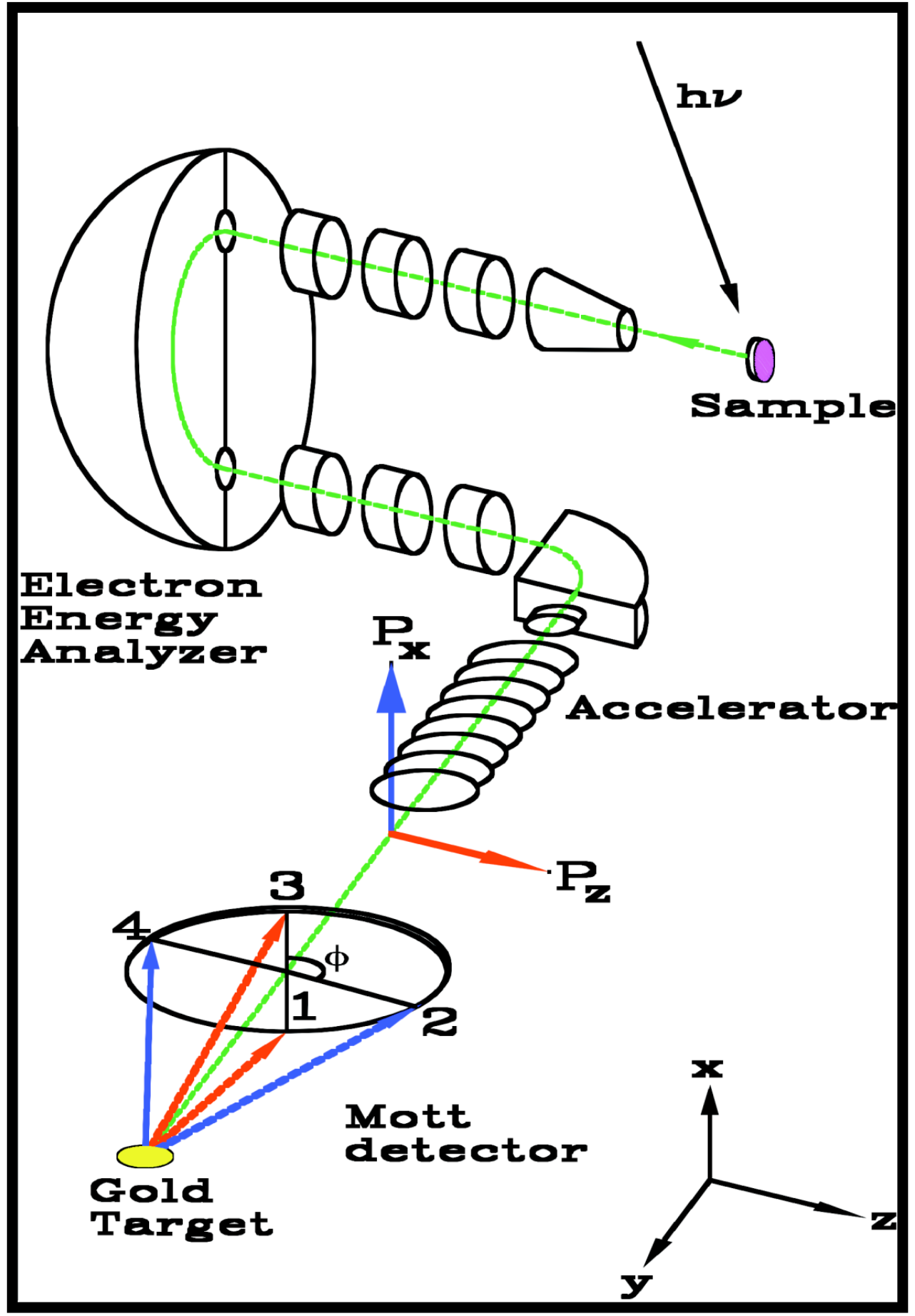

Figure 1

Experimental layout. See text for details.

Tobin, LLNL 


\section{Investigations of Magnetic Overlayers at the Advanced Photon Source}
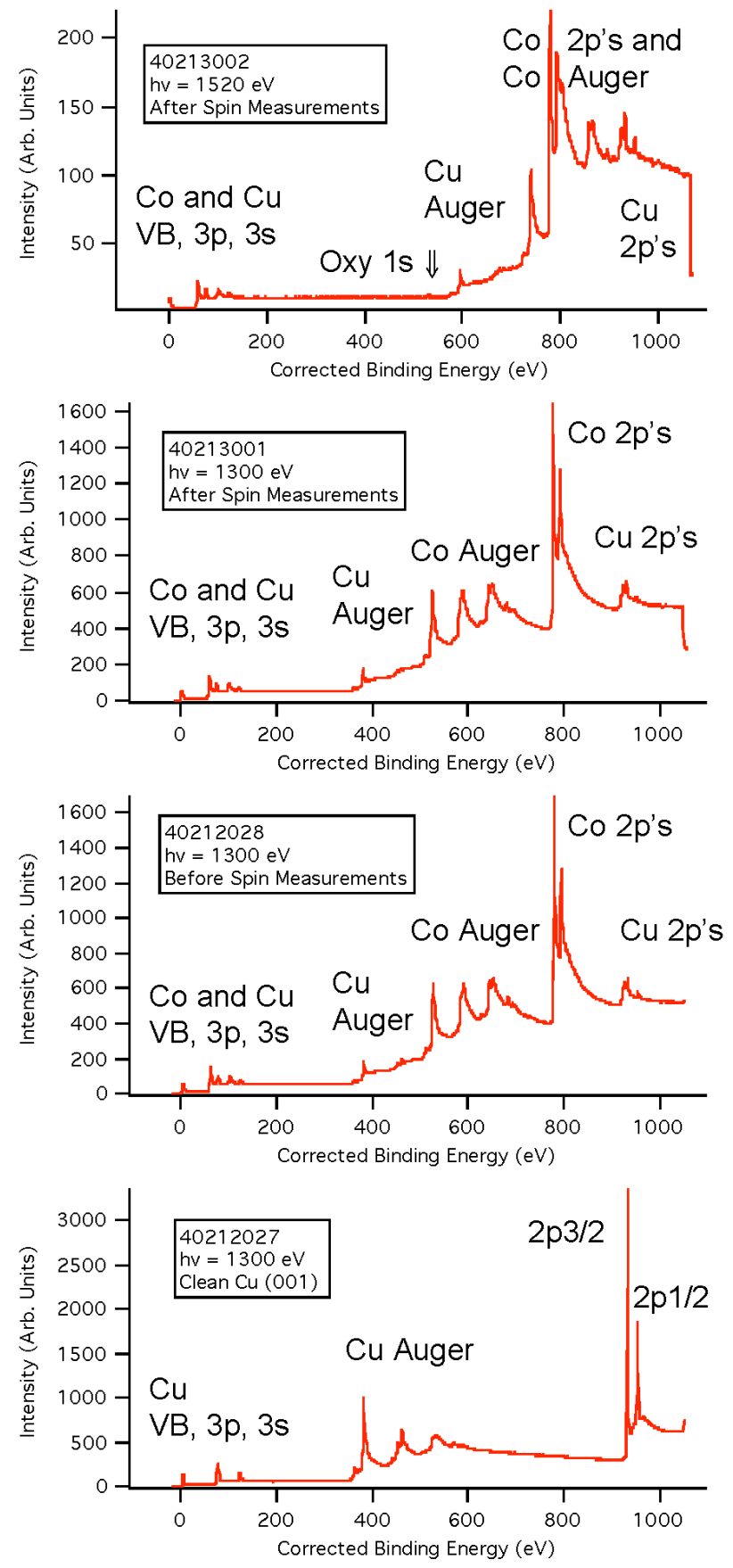

Co Spin Exp-Wide Scans

27Feb08

Data from Feb 2004

Nominal Photon Energies

$1250 \mathrm{eV}$ (MgKa) and

$1450 \mathrm{eV}$ (AlKa)

Corrected Photon Energies

Approximately $1300 \mathrm{eV}$

And $1520 \mathrm{eV}$

Nominal Coverage

$10 \mathrm{ML} \mathrm{Co} / \mathrm{Cu}(001)$

Plot widths are from

-100 to $1100 \mathrm{eV}$ BE

Figure 2

Wide PES or X-ray Photoelectron Spectroscopy (XPS) scans of $\mathrm{Co} / \mathrm{Cu}(001)$ using hv $=1300$ and $1520 \mathrm{eV}$.

Tobin, LLNL

Page 7

$6 / 25 / 09$

J. Vac. Sci. Tech., AVS International Symposium, San Jose, CA, USA, 2009 


\section{Investigations of Magnetic Overlayers at the Advanced Photon Source}

Fe Spin Exp-Wide Scans

Data from Feb 2005
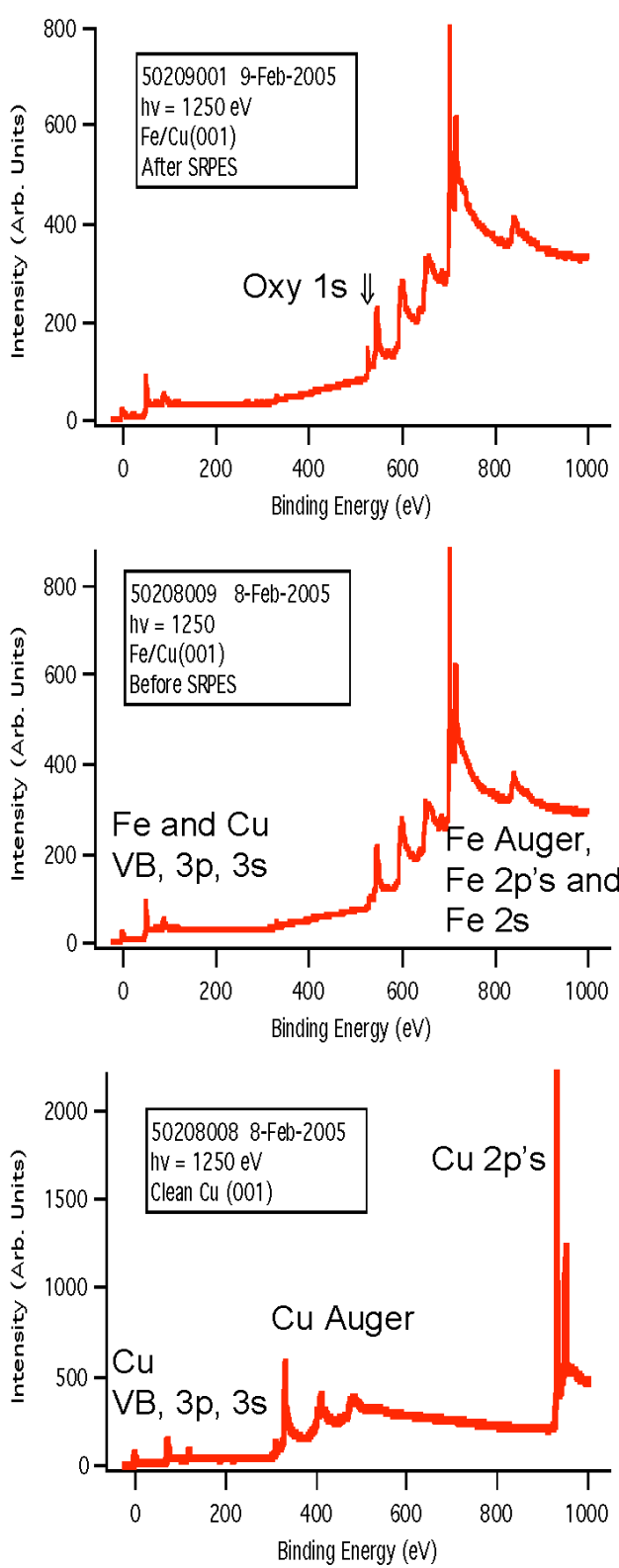

29Feb08
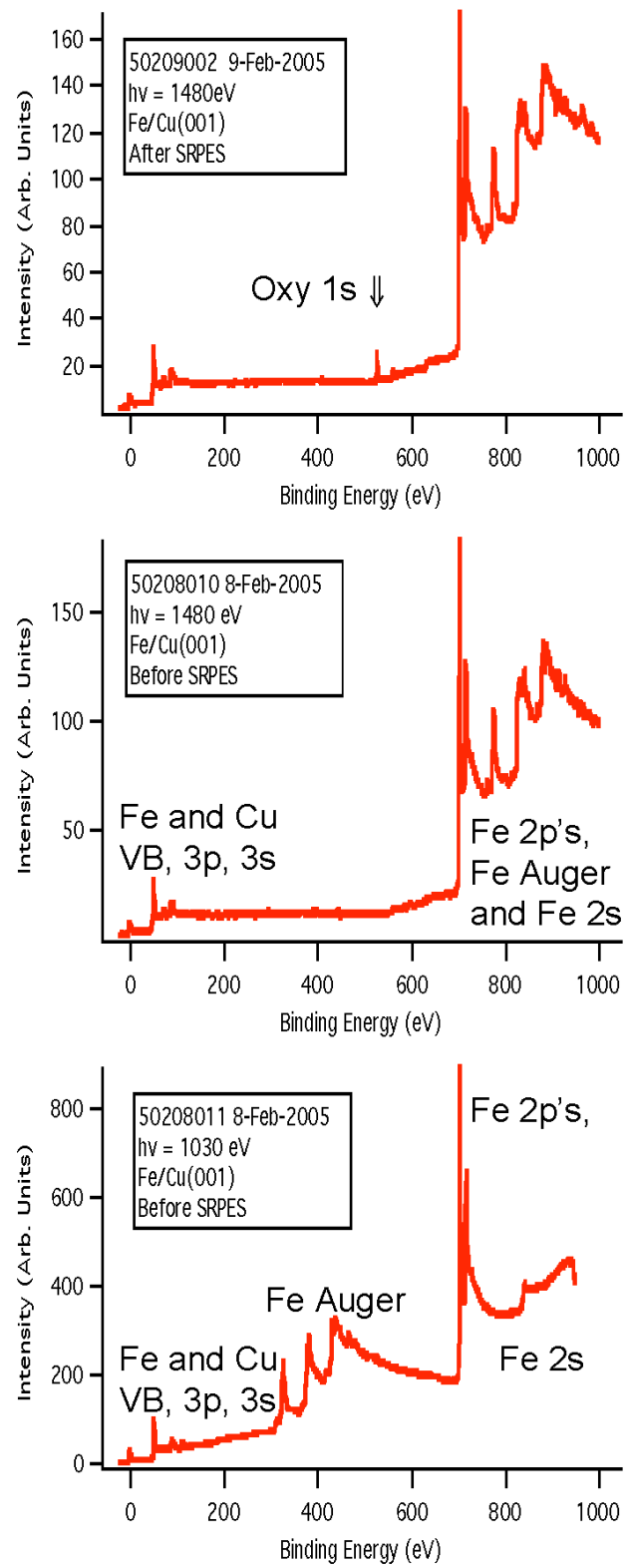

Figure 3

Wide PES or XPS scans of $\mathrm{Fe} / \mathrm{Cu}(001)$ using hv $=1250$ and $1480 \mathrm{eV}$.

Tobin, LLNL 


\section{Investigations of Magnetic Overlayers at the Advanced Photon Source}

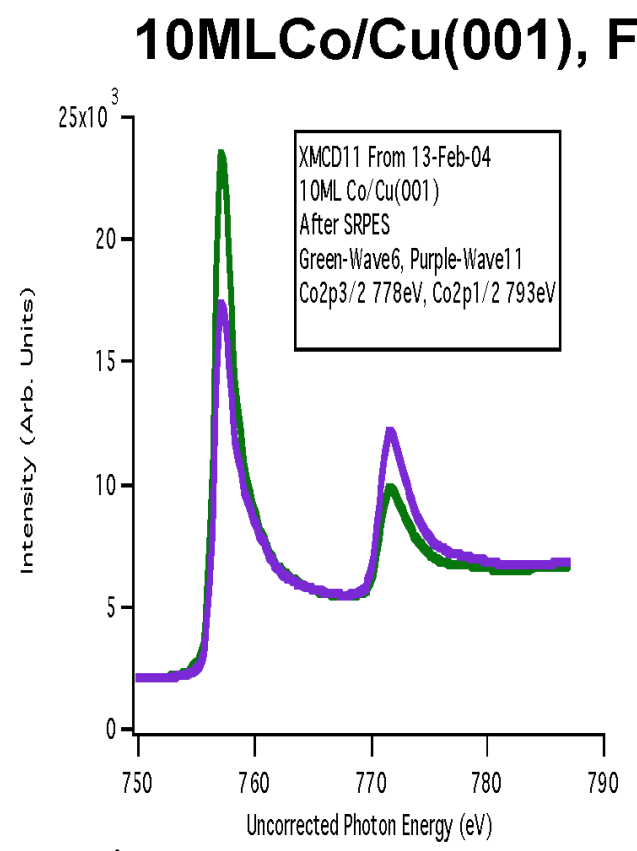

eb 2004, XMCD-Abs
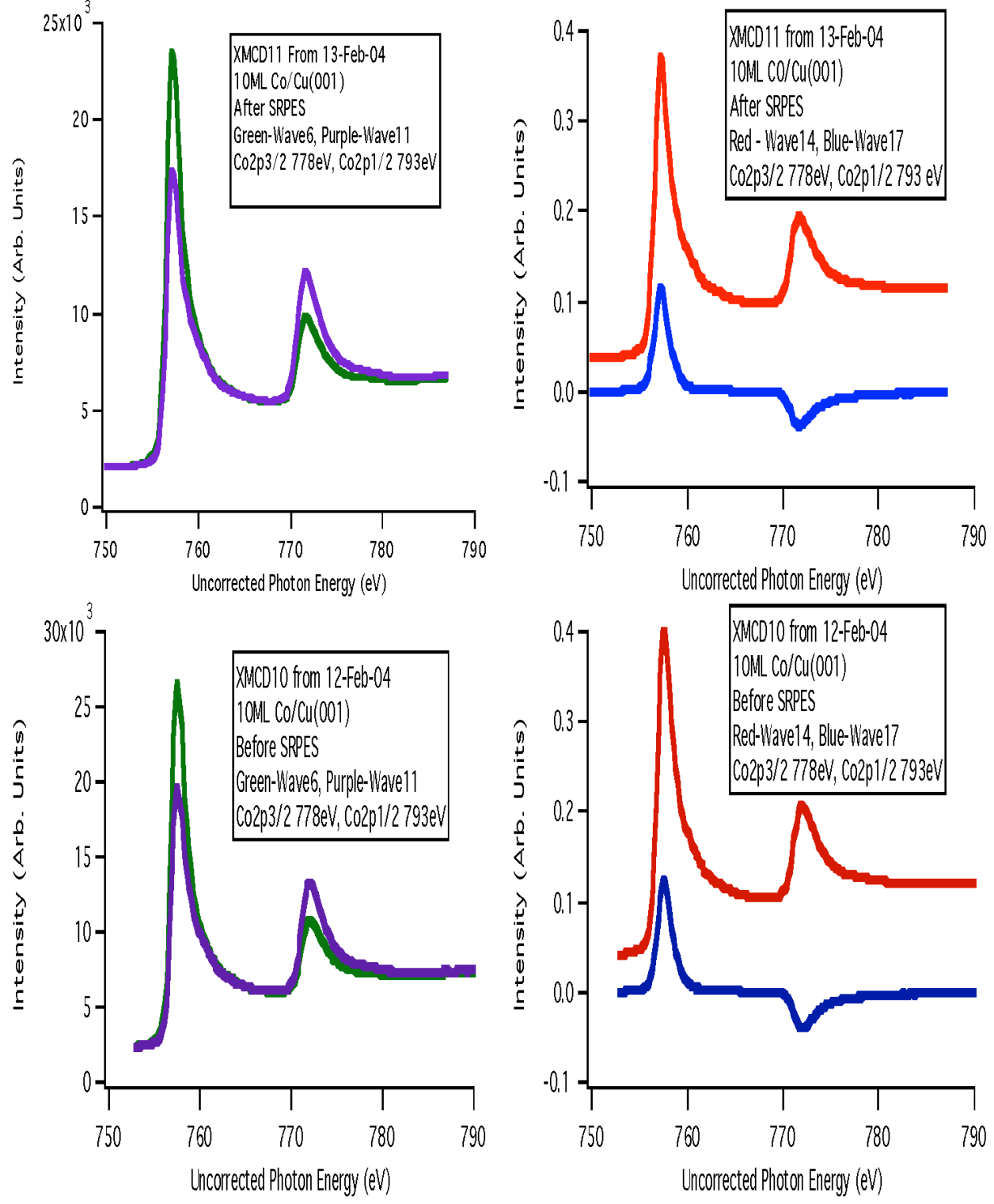

Figure 4

XMCD-Abs of $\mathrm{Co} / \mathrm{Cu}(001)$. The plots in the left column show the "raw" spectra, with magnetization reversal or helicity reversal. The right column includes plots of the corresponding sums (red) or differences (blue). The Bottom and Top correspond to before and after the various SRPES measurements, respectively.

Tobin, LLNL

Page 9

$6 / 25 / 09$

J. Vac. Sci. Tech., AVS International Symposium, San Jose, CA, USA, 2009 


\section{Investigations of Magnetic Overlayers at the Advanced Photon Source}

\section{$\mathrm{Fe} / \mathrm{Cu}(001)$, Feb 2005, XMCD-Abs}
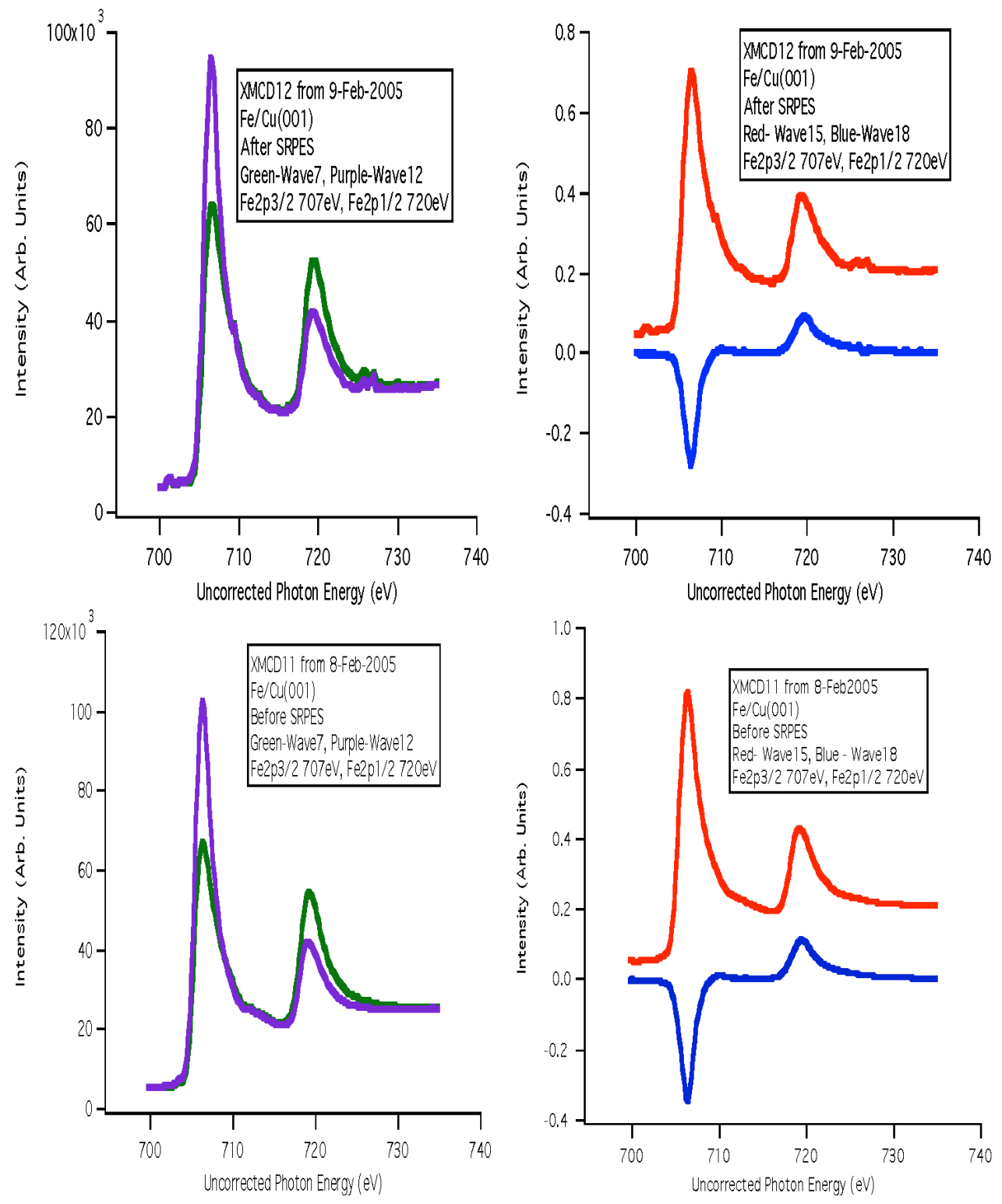

Figure 5

XMCD-Abs of Fe/Cu(001). The plots in the left column show the "raw" spectra, with magnetization reversal or helicity reversal. The right column includes plots of the corresponding sums (red) or differences (blue). The Bottom and Top correspond to before and after the various SRPES measurements, respectively.

Tobin, LLNL

Page 10

$6 / 25 / 09$

J. Vac. Sci. Tech., AVS International Symposium, San Jose, CA, USA, 2009 


\section{Investigations of Magnetic Overlayers at the Advanced Photon Source}

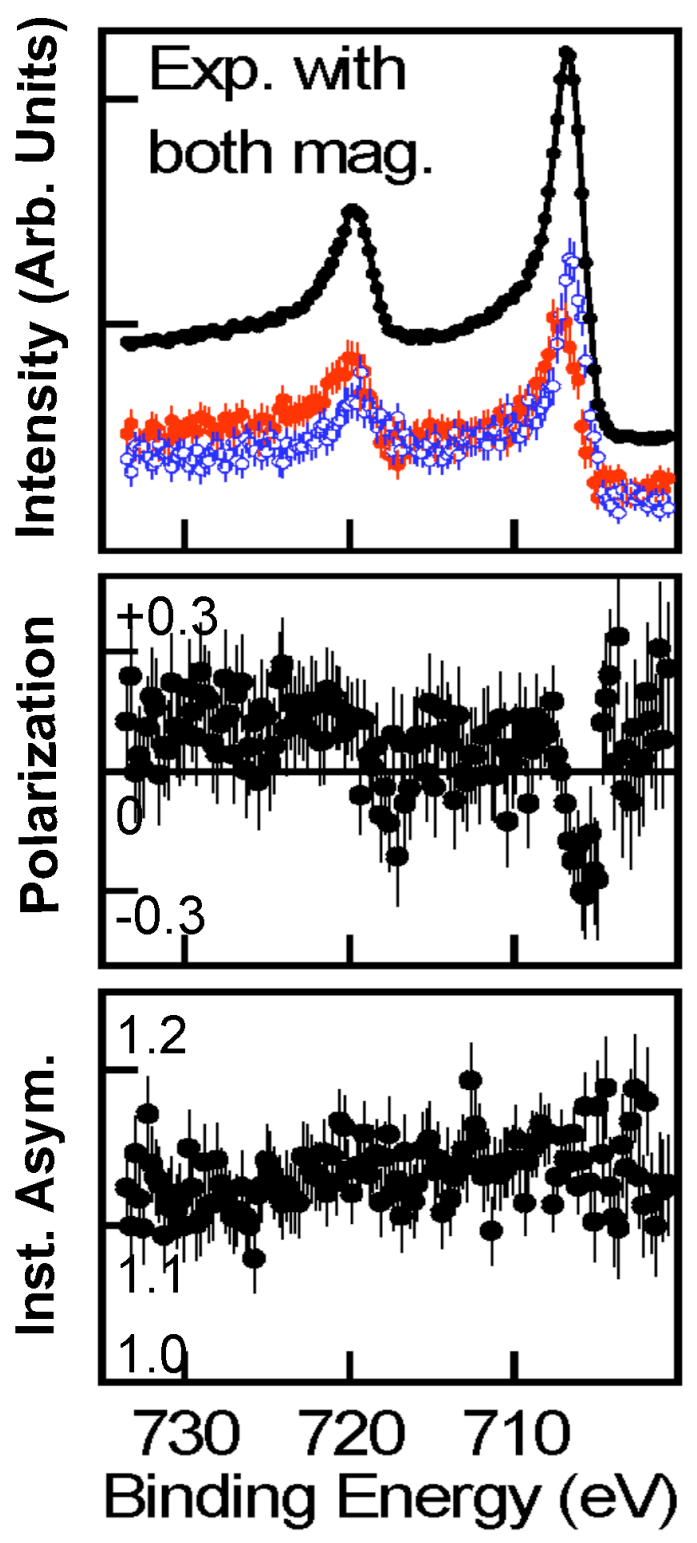

Figure 6

Spin resolved photoelectron spectroscopy (SRPES) of macroscopically magnetized Fe films. From the top, the following are shown: Experimental spectra, polarization and instrumental asymmetry. $[7,10]$ Here, the axis of quantization is along the $+/-x$ direction, following the magnetization. The photon energy was $1030 \mathrm{eV}$, with right circular polarization. 


\section{Investigations of Magnetic Overlayers at the Advanced Photon Source}
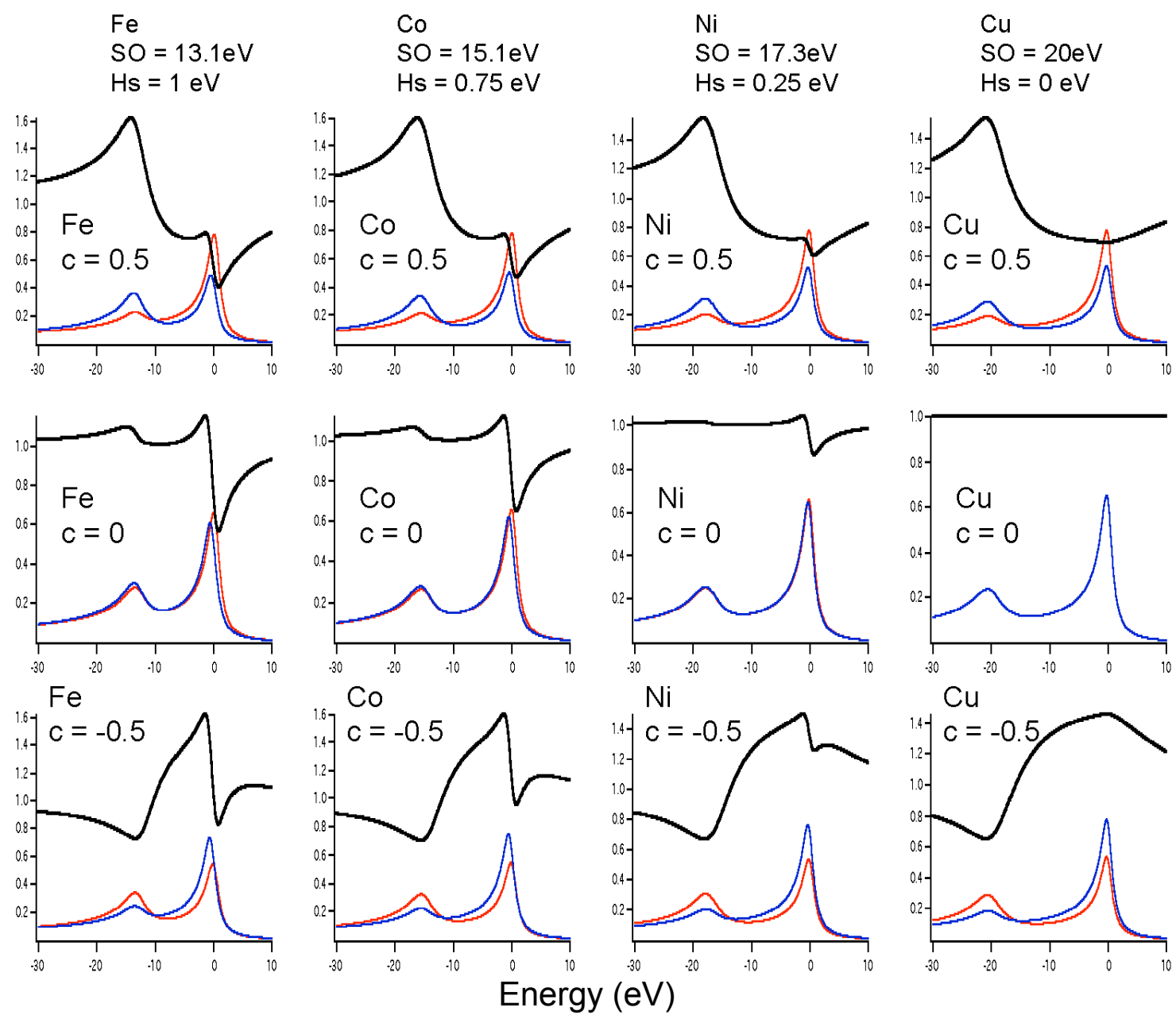

Figure 7

The simulated spectra for an idealized configuration and a number of limiting cases, using our single electron model. [7,11] Spin up is red. Spin down is blue. The ratio of the spin down to spin up is shown as a black line. To avoid divergence, the ratio was calculated as ratio $=[$ l (spin down) + small constant $]$ / [I (spin up) + small constant]. Three dichroism cases were considered: $c=0.5$ (strong positive); $\mathrm{c}=0$ (no dichroism); $\mathrm{c}=-0.5$ (strong negative dichroism). Four spin orbit cases were utilized, corresponding to $\mathrm{Fe}, \mathrm{Co}, \mathrm{Ni}$ and $\mathrm{Cu}$. SO is the effective spin orbit splitting of the $2 p 3 / 2$ and $2 p 1 / 2$ levels. [12] Hs is the exchange splitting, obtained in part from scaling from the magnetic moments of each element. $[5,7]$ 